\title{
Universidad, entre poiesis y politeia ${ }^{1}$
}

\section{University, among poiesis and politeia}

\author{
Fabián Alonso Pérez Ramírez ${ }^{2}$ \\ Universidad Católica de Oriente, Colombia \\ Juan Carlos Franco Montoya ${ }^{3}$ \\ Universidad Católica de Oriente, Colombia
}

Recepción: 10 de junio del 2018

Evaluación: 4 de agosto del 2018

Aceptación: 12 de septiembre del 2018

1 Este artículo es derivado de la investigación doctoral titulada: La Otra Universidad en Colombia. Adscrita al grupo de investigación: Servicio Educativo Rural (SER) de la Facultad de Ciencias de la Educación, Universidad Católica de Oriente (Rionegro, Antioquia). Agradecemos el respaldo ofrecido por la Dirección Académica, la Dirección de Investigación y Desarrollo y el Comité Institucional de Cualificación y Evaluación del Personal (CICEP) de la Universidad Católica de Oriente.

2 Candidato a Doctor en Ciencias de la Educación por la Universidad de San Buenaventura, sede Medellín ( $1^{\circ}$ cohorte) con la investigación: La Otra Universidad en Colombia. Magíster en Educación, Especialista en Pedagogía y Didáctica, Licenciado en Filosofía y Ciencias Religiosas de la Universidad Católica de Oriente (UCO). Con estudios en Artes Plásticas de la Escuela Popular de Arte (EPA). Docente asistente adscrito a la Universidad Católica de Oriente. Correo electrónico institucional: fperez@uco.edu.co

3 Candidato a Doctor en Educación por la Universidad Internacional Iberoamericana de México, Magíster en Educación y Licenciado en Matemáticas de la Universidad Católica de Oriente. Docente asociado de la Universidad Católica de Oriente. Correo electrónico institucional: jfranco@ uco.edu.co 


\title{
Resumen:
}

Este artículo pretende plantear dos ideas: la primera problematiza el origen de la universidad en tanto creación humana en sentido pragmático (Kant, 1800) para luego abordarlo en perspectiva histórica desde tres momentos: Medieval, Clásico y Ancestral retomando autores como Rashdal (1895), Haskings (1923), Ureña (1919), Ribeiro (1971) y Dussel (2004). Posteriormente se plantea el origen de la universidad más allá de su historicidad, en el interior del ser humano como creación erótica de cultivo de la virtud y el conocimiento poiesis en Platón (380 a.C/1999). La segunda idea implica comprender que desde su origen la universidad como politeia, y a partir de la apropiación de algunos planteamientos aristotélicos (s,f/1883) y arendtianos (1997) ha posibilitado pensarse como un escenario de movilización social o continuidad del orden establecido, donde se reflexiona en la necesidad de actualización de la universidad entre poiesis y politeia como potencia para recuperar su sentido primigenio (filosófico) en tanto ese esfuerzo espiritual y colectivo de la humanidad por alcanzarse a sí misma.

Palabras-Clave: universidad, filosofía, virtud, conocimiento, política.

\begin{abstract}
:
This article pretends to establish two ideas: the first proposes the problem of the origin of the university as human creation in the pragmatic sense (Kant, 1800), then we approached to it in an historical perspective considering three moments: Medieval, Classic, and Ancestral. Some authors as Rashdal (1895), Haskings (1923), Urena (1919), RIbeiro (1971), and Dussel (2004) are considered here. Afterward, it is proposed the origin of the university beyond its historicity, in the inward of the human being as an erotic creation of the culture of the virtue and knowledge, poiesis of Platon (380 BC / 1999). The second idea requires to comprehend the university from its beginning as a politeia, and from the appropriation of some Aristotelian (sf / 1883) and Arendthian (1997), statements, it has been able to think of it as a scenario of social movilization or perpetuation of the established order. It is there that we saw the need for an update of the university between poiesis and politeia, as a strength to recover its primal sense (a philosophical one) from the spiritual and collective effort of the humankind to reach herself.
\end{abstract}

Keywords: university, philosophy, virtue, knowledge, politics. 


\section{Université, entre Poiesis et Politeia}

\section{Résumé:}

Cet article tente de présenter deux idées : la première problématise l'origine de l'université en tant que création humaine dans un sens pragmatique (Kant, 1800) pour ensuite l'aborder dans une perspective historique à partir de trois moments : médiéval, classique et ancestral, en reprenant es auteurs tels que Rashdal (1895), Haskings (1923), Ureña (1919), Ribeiro (1971) et Dussel (2004). Puis, on présente l'origine de l'université au-delà de son historicité, à l'intérieur de l'être humain comme création érotique de la culture de la vertu et de la connaissance poiesis chez Platon (380 a.C/1999). La deuxième idée implique de comprendre que, dès son origine, l'université en tant que politeia, et à partir de l'appropriation de certaines approches aristotéliciennes (s,f/1883) et arendtiennes (1997), a permis de la penser comme scénario de mobilisation sociale ou de continuité de l'ordre établi, où l'on réfléchit à la nécessité d'actualisation de l'université entre poiesis et politeia, comme une puissance pour retrouver sa signification originale (philosophique) en tant que cet effort spirituel et collectif de l'humanité pour s'auto-atteindre.

Mots-clés: université, philosophie, vertu, connaissance, politique

\section{Universidade, entre poiesis e politeia}

\section{Resumo:}

$\mathrm{O}$ artigo tenta propor duas ideias: a primeira problematiza a origem da universidade como criação humana no sentido pragmático (Kant, 1800) para logo, abordar uma perspectiva histórica desde três momentos: medieval, clássico e ancestral retomando autores como Rashdal (1895), Haskings (1923), Ureña (1919), Ribeiro (1971) e Dussel (2004). Posteriormente, se propor a origem da universidade além da historicidade, no interior do ser humano como criação erótica de cultivo da virtude e do conhecimento poiesis em Platão (380 a.C/1999). A segunda ideia implica compreender que desde a origem a universidade como politeia, e apartir da apropriação de algumas propostas aristotélicas (s,f/1883) e arendtianos (1997) como possibilidade de pensar uma cena de mobilização social ou continuidade da ordem estabelecido, que reflete na necessidade de atualização da universidade entre poiesis e politeia como potência para recuperar seu sentido primigênio (filosófico) entanto esse esforço espiritual e coletivo da humanidade se alcança a si mesma.

Palavras-chave: universidade, filosofia, virtude, conhecimento, política. 


\section{Introducción}

Cuando decimos universidad, en primera instancia, estamos hablando de una creación humana. A simple vista, está claro que no han creado universidades los otros reinos de la naturaleza (mónera, protista, fungi, micota, animalia), lo cual nos conduce directamente a una cuestión antropológica, pues, aunque pertenezcamos al reino animalia -es decir, pese a compartir características físicas y anímicas con los reptiles, mamíferos y, sobre todo, con nuestros antepasados, los homínidos bípedos- no es ésta nuestra única naturaleza. Kant, al respecto, afirmaba:

Una ciencia del conocimiento del hombre sistemáticamente desarrollada (Antropología), puede hacerse en sentido fisiológico o en sentido pragmático. -El conocimiento fisiológico del hombre trata de investigar lo que la naturaleza hace del hombre; el pragmático, lo que él mismo, como ser que obra libremente, hace, o puede y debe hacer, de sí mismo (1991, p.7)

Es así como la naturaleza (physis) obra en lo humano en tanto animal (zoon), fisiológicamente hablando, mientras que lo humano no solo obra en la naturaleza (poiesis) dialécticamente, sino que lo humano obra también sobre lo humano mismo, he ahí su otra naturaleza (politeia). Lo humano se hace entre humanos, hoy más que nunca, dado que "la Modernidad, entre sus tantas acepciones, es la época en la que lo humano se forma a partir del hombre mismo" (Hincapié, 2016, p. 258).

Este hombre que obra libremente sobre sí mismo, al determinar su sentido pragmático, su naturaleza cultural, su posibilidad abierta al mundo, se forma en relación con otros hombres; $y$, dada su naturaleza inconclusa, en esa interacción con los otros, determina su naturaleza y trans-forma su entorno al crear. Por supuesto, una de esas creaciones humanas es la universidad. Abordemos cómo esta creación humana presenta una historicidad que se disputa tres perspectivas de enunciación, para luego asumir la universidad en tanto poiesis y politeia.

El origen histórico de la universidad confronta tres perspectivas, a saber: medieval, clásica y ancestral. De acuerdo con Rashdall (1895, p. 144) y Soto (2007, p. 404), la universidad, como institución formal, tiene su origen en la Europa medieval del siglo XII, y encuentra su enunciación en el cuño

$\overline{4}$ Las cursivas pertenecen al autor citado. 
de los términos universitas magistrorum et escholarium. Se trata de una corporación o agrupación, de un cuerpo colegiado de maestros y estudiantes alrededor del saber, que para la época aparece con las facultades de teología, medicina y derecho en las primeras universidades (Sorbona, Bolonia, Oxford, Salamanca).

Sea cual fuere el origen de la universidad, está claro que el contexto de la universidad como institución social en esta génesis se identifica a partir de unas características fundamentales: la agremiación de estudiantes y maestros alrededor de los saberes, la búsqueda de una visión universal e integradora, la consolidación y organización de los saberes que luego se reflejarían en la organización de los currículum, la formación teórica y técnica de avanzada, la autonomía universitaria en términos legales (vía las cédulas reales y las bulas papales) y en el sentido intelectual esto configuró un nuevo tercer poder (entre el papado y las monarquías), vía el uso de la razón con sus dominios y su relación con el poder en tanto la formación de élites dirigentes que posibilitaron además, según Soto (2007), la transición de los llamados oficios a la consolidación de profesiones y profesionales, entre ellas, la del saber enseñable o los magistrorum, que produjeron teólogos, abogados, médicos, en estas primeras facultades que acompañaron el surgimiento de las universidades (Pérez y Ríos, 2017b, p. 7).

Se podría decir que estas primeras universidades europeas presentaban una relación directa con los poderes hegemónicos de la Edad Media, el papado y la monarquía, a tal punto que para poder funcionar necesitaban de la bula papal (vía el clero) o la cédula real (vía la monarquía). Además, hay una situación que da cuenta de la capacidad de movilización social que la universidad experimentó; muchos de los oficios se convirtieron por fuerza de la universidad en profesiones y los textos escritos de las facultades anteriormente nombradas se convirtieron en fuentes de poder, tal es el caso de la Biblia, el vademécum y los diferentes códigos.

Otra perspectiva histórica reclama el origen de la universidad en las escuelas filosóficas de la Grecia clásica. Ureña (1919, p. 60), por ejemplo, señala que en la Academia de Platón y en el Liceo de Aristóteles es posible encontrar la ruta formativa que daría origen, en la Edad Media, a las facultades de artes y a los estudios generales previos (studia generalia) requeridos para cursar las carreras de teología, derecho y medicina. Dicha ruta formativa configuró el trivium (dialéctica, gramática y retórica) y el quadrivium (matemática, geometría, astronomía y música), esto es, el conjunto de estudios generales que se impartía en todas las universidades medievales. 
Hay que decir, además, que los métodos de enseñanza o "las estrategias que hasta nuestros días acompañan los procesos de formación universitaria por medio de temas fijos o improvisados, las conferencias, los debates, las lecturas y los comentarios, la escritura de textos donde sintetizaban sus enseñanzas" (Pérez et al, 2015, p. 112), fueron creados y desarrollados por los sofistas de la Grecia antigua.

Y una tercera perspectiva de enunciación del origen de la universidad está dado en contra vía del eurocentrismo, al posicionar su origen en civilizaciones asiáticas e indias que permitieron configurar conocimientos teóricos $\mathrm{y}$ prácticos acumulables por la potencia de las comunidades que a su vez eran transmitidos de generación en generación (Ribeiro, 1971, p. 58) y (Dussel, 2006, p. 36); Las escuelas de Nanjing (China, siglo III a.C.), Nalanda (India, siglo V d.C.) y las escuelas judías de los Gueonim (siglo VII d. C.) ofrecen pruebas palpables.

Pero valdría la pena detenerse en las implicaciones que se derivan de lo humano como poiético y politéico, con el fin de ver de qué manera determinan a una de sus creaciones: la universidad. Ante todo, se trata de establecer cómo la universidad tiene la potencia de ser poiética y politéica al mismo tiempo.

\section{Universidad como poiesis}

En el diálogo El Simposium, o de la Erótica, Diotima discurre con Sócrates y los presentes sobre la capacidad de creación humana (poiesis) en relación con lo que es el amor. En ese contexto, el agape y la poiesis son concebidos como una búsqueda de la inmortalidad humana; búsqueda que, como se verá, implica una politeia para cada uno de los niveles de la poiesis desarrollados en este diálogo, los cuales nos servirán como matriz de comprensión de la universidad, en tanto esfuerzo por alcanzar la inmortalidad de lo humano.

El concepto de $\pi$ oínбıৎ es algo muy amplio, ya que ciertamente todo lo que es causa de que algo, sea lo que sea, pase del no ser al ser es "creación", de suerte que todas las actividades que entran en la esfera de todas las artes son creaciones y los artesanos de estas, creadores (Platón, 1999, p. 83).

Siguiendo con el diálogo, Diotima plantea que existen tres acciones perseguidas por los seres humanos en ese afán de inmortalidad que posibilitan la poiesis. Una de ellas es la acción de procrear "porque es la generación algo eterno e inmortal, al menos en la medida en que esto puede darse en un mortal" (Platón, 1999, p. 86). 
La procreación es el primer eslabón de lo humano en relación con su preservación y necesidad para dar continuidad a la especie, ya que ésta requiere generarse a sí misma para perpetuarse. Dicha generación es poiesisphysis al mismo tiempo; no obstante, ya presenta visos de politeia, en la medida en que el vínculo de lo humano para la procreación, de por sí, implica una relación íntima, no solo sexual, sino erótica, que, en medio del deseo y el amor, se proyecta en tanto creación de lo humano como obra y perpetuación del amor erótico, de los dos que se unen y crean otro ser desde ellos mismos.

Sin embargo, se ha de tener en cuenta también que no toda creatura en potencia engendrada es fruto del amor erótico y relacional humano que le daría su sentido más allá de lo puramente sexuado, sino que, en otros casos, se da por la relación sexual y voluptuosa que no compromete en profundidad la condición humana, lo que, como se ha visto, degenera lo humano mismo y crea tantas dificultades en el cuidado y crianza de ese nuevo ser.

La segunda acción poiética, según Diotima, es la creación de ciudades (polis) y "dejar para el futuro una fama inmortal" (Hexámetro citado por Platón, 1999, p. 89). Se refiere así al ideal de los héroes que alcanzan la inmortalidad por medio de la memoria del pueblo, sin necesidad de procreación. En este punto, se encuentra una relación entre poiesis y politeia cada vez más amplia, en tanto implica un relacionamiento no solo en la esfera natural, erótica, sino también social.

La fama que inmortaliza está presente en la capacidad que el ser humano tiene de llevar hasta sus más altos niveles sus potencialidades, al marcar un lugar inédito que logra inmortalizarlo a través de la memoria de los habitantes de la ciudad. Implica vencerse a sí mismo, para luego, y dado el caso de la puesta en escena de las destrezas, vencer a los otros, según lo que se ponga en juego, para alcanzar la inmortalidad social.

Y la tercera acción poiética de lo humano es el cultivo de la virtud y el conocimiento, que pone lo humano en contacto con la vida contemplativa (bios teoretikós). Esta tercera acción obra en lo humano como impulso de inmortalidad, en la que se plantea un origen interno (erótico-creativo) de la universidad en tanto poiesis, actitud humana de creación, búsqueda y compromiso con la virtud y el conocimiento.

Que la universidad tenga un origen más allá del debate histórico, en el interior del ser humano, en tanto poiesis (creación erótica) o búsqueda de la virtud y 
el conocimiento, supone además un vínculo especial con la politeia, ya que la virtud y el conocimiento implican el relacionamiento consigo mismo, con los demás y con la posibilidad de movilizar socialmente la naturaleza humana, esto de por sí es conflictivo.

Es necesario entonces des-ocultar (como la aletheia) lo que es la virtud, el conocimiento y sus implicaciones como acciones poiéticas de lo humano que -en su sentido interno- procuraron el surgimiento de la universidad mucho antes de su institucionalización lograda en las altas escuelas. Lo que se busca es poner de relieve el espíritu de la universidad como actitud de apertura interior virtuosa (ético-estética) y racional (epistemológica).

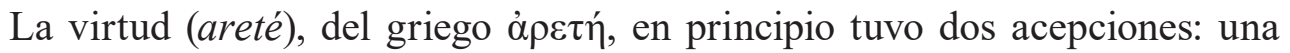
referida a la eficacia y fuerza de alguien o de algo; otra para dar cuenta de la excelencia que se alcanza (Platón, 1993, p. 25). Posteriormente, la areté implicó el cultivo de cualidades físicas, éticas, estéticas, intelectuales y espirituales, conquistadas por el arduo trabajo de lo humano sobre sí mismo.

Platón dirá que "nuestra ciudad si está rectamente fundada, será completamente buena (...) será prudente, valerosa, moderada y justa" (1993, p. 177). De estas cualidades surgieron posteriormente las virtudes cardinales. Ahora bien, lo que dice Platón sobre la fundación de las ciudades puede ser cuestionado, dado que los hechos muestran que el surgimiento de estos centros urbanos muchas veces fue el resultado de la imposición de unas fuerzas colectivas humanas sobre otras. De acuerdo con W. Benjamin (1991, p. 5), las fuerzas vencedoras instauran un nuevo orden que se legitima a partir de un marco normativo, lo que viene a representar otra forma de violencia.

De igual manera, podríamos pensar que la fundación y preservación de toda universidad implica siempre luchas, conflictos internos de los sujetos por vencerse a sí mismos (pasajes entre la virtud y el vicio, el conocimiento y la ignorancia). Además, las formas de organización de la misma universidad son una lucha permanente entre los cuerpos colegiados, sus intereses, los saberes, el poder y su relacionamiento con otras instituciones, como el Estado, los gremios productivos, lo jurídico, la política, entre otros.

Es así como la prudencia, el valor, la moderación y lo justo responderían a ese ideal formativo de lo humano (1993, p. 179) en tanto posibilidad de alcanzar niveles superiores de sí mismo. Estas virtudes se alcanzarían como ejercicio y conquista permanente, como condición y ejercitación de la 
universidad entendida como actitud interior erótico-creativa, mucho antes de su institucionalización y realización material.

Entonces, ejercitar la prudencia por la razón es la ciencia de la preservación, del buen consejo, la eubolia ${ }^{5}$ pitagórica. El valor resulta de la constancia de la recta opinión sobre las cosas que se han de temer, opinión (en su mejor caso conocimiento) prescrita por el legislador, que hace del valor también obediencia. En este sentido, no solo por la normalización externa que hace el legislador (para el caso de la universidad, internamente el docente, decano, incluso el estudiante; pero también desde la exterioridad normativa internacional y nacional), sino y, además, la obediencia a sí mismo como posibilidad de autorregulación. Aquí el rasgo de la politeia acontece como posibilidad de hacerse cargo de sí mismo en tanto autogobierno y no solo en tanto condición externa de relacionamiento con los otros.

La templanza es tanto orden y dominio de placeres como concupiscencia, ser dueños de símismos (Platón, 1999, p. 184), es moderación del carácter indómito. Y la justicia no es mera compensación de daños, ni mucho menos servirse de los propios intereses para actuar y juzgar según un poder o condición; por el contrario, es un modo de servir a los intereses de la comunidad comunitas, de la polis, de la universitas, que hace posible la felicidad, el bien común de los hombres, como en su sentido primigenio de corporación.

Así, tendríamos que preguntarnos si este cultivo de las virtudes -la prudencia, el valor, la moderación y la justicia (y su consecuente felicidad) - son hoy en día estandarte de la universidad del siglo XXI, incluso si han sido de la universidad medieval, renacentista, ilustrada, moderna, contemporánea. Además, valdría la pena preguntarse si esto responde a un olvido de la universidad en sí misma, en la medida en que uno de los signos de nuestro tiempo es la falta de la pregunta por la idea de universidad e incluso su silencioso desplazamiento y reemplazo por la aparición de las Instituciones de educación superior. Esta tesis ha de problematizarse en relación con los principios fundantes y orientadores de la universidad en el mundo, en toda época y en cualquier lugar, como horizonte y retorno posible de re-creación humana y recuperación eidética.

5 Del griego ev̉ßov $\lambda i ́ \alpha$, que convoca a hablar con prudencia. Valor promovido por los pitagóricos en sus ejercicios de purificación y expiación del alma. 
Dicha recuperación eidética se da por un doble olvido al que la idea de universidad ha sido sometida en nuestro tiempo: por un lado, la condición cesante de una realidad que pudo ser y no es en el presente continuo al que asistimos en este vertiginoso siglo XXI, por su inadvertido y silencioso derrumbamiento en tanto idea de universidad, dada la pérdida de su naturaleza (que es la pérdida de su origen erótico, más allá de su origen histórico) y la instauración inusitada de su desplazamiento, al ser reemplazada por Instituciones de educación superior. Por otro lado, el olvido se manifiesta en la ausencia de la pregunta que indaga por la universidad, que supone la superación de la inquietud en la naturalizada afirmación que reza solo si y en tanto que Educación Superior.

Este doble olvido de la idea de universidad es un signo de este tiempo que nos ha tocado vivir: el de la institucionalidad entendida como una forma de gubernamentalidad, de control de la población determinada por las relaciones de saber-poder, que establece la producción de sujetos para los intereses de la economía política (Foucault, 2015, p.35) y deja de lado la formación para imponer sigilosamente la semiformación (Nietzsche, 1872/2000, p. 179); Adorno, 1959, p. 151). En suma, el desplazamiento de la formación (Bildung) por la semiformación (Halbbildung) es el desplazamiento de la idea de universidad por la materialización de las instituciones de educación superior.

De esto se sigue que las instituciones de educación superior del siglo XXI estén alineadas con "las lógicas funcionalistas de acreditación estandarizada impuestas por el gerencialismo consumista" (Pérez y Ríos, 2017a, p. 84), que generan un mercado académico capaz de producir "el discurso no universitario de la universidad" (Naishtat, García, Villavicencio, 2001, p. 8). Con esto se problematiza tanto su autonomía como su razón de ser, dado que desde sus orígenes están sometidas al "control jurídico" y a la ya gastada expresión de las crisis (administrativas, presupuestales, curriculares y profesionales). La decadencia de la idea de universidad y del triunfo de las Instituciones de educación superior repercute en el crecimiento de las desigualdades sociales, al profundizar su adaptación y transfiguración, y al debilitamiento de la capacidad reflexiva, crítica y de acción ${ }^{6}$.

\footnotetext{
6 De acuerdo con C. Restrepo, "esta destrucción, empero, tiene que ver mucho más, que con el hecho de que la universidad contemporánea haya perdido su soberanía y su libertad, al ser ocupada por intereses extracognitivos" (2013, p. 84).
} 
La idea de universidad, entendida como el esfuerzo espiritual y colectivo de la humanidad por alcanzarse a sí misma, como un medio para la perfectibilidad del hombre, se desdibuja en la bruma del tiempo que sigue caminando en medio de las ruinas de una época que no la vio nacer, mucho menos crecer y que al mirar hacia atrás la encontró lejana, goteando con brillo tembloroso, desafiando su destino en un ejercicio de recuperación eidética, de recuerdo y actualización permanente constituyente de la universitas en tanto formación de lo humano.

Así, el cultivo del conocimiento poiético en la universidad implica por lo menos que busca la episteme y no la simple opinión (doxa). Esto es, que no basta quedarse en el thaumazein - "el pasmo ante el milagro del ser (...) comienzo de toda filosofía" (Arendt, 1993, p. 327) - o frente al vertiginoso siglo que nos asiste, sino que se hace necesaria la inquietud permanente y la elaboración de un método (un camino) para ir hacia su descubrimiento, hacia su frontera comprensiva, hacia sus límites.

El cultivo de la virtud y del conocimiento - como actitud interior, creación erótica y como disposición de lo humano hacia la naturaleza de las cosas y de lo humano mismo- pone de antemano frente a la condición humana el surgimiento de la universidad. Se trata de un acontecimiento íntimo de relacionamiento consigo mismo (nai idio), con la virtud, la naturaleza (physis) y la vida política (bios politikós). Aproximémonos entonces a la universidad en clave politéica.

\section{Universidad como politeia}

La universidad, en tanto politeia, implica la condición gregaria de lo humano; "la naturaleza arrastra, pues, instintivamente a todos los hombres a la asociación" (Aristóteles, 1873, p. 26). Así mismo, la universidad -en su sentido primigenio- es una asociación: "universitas significaba una comunidad o asociación, un colegio o cuerpo constituido con miras a un fin determinado" (Soto, 2007, p. 401). Pero dicha condición gregaria no es suficiente para comprenderla en tanto politeia, por lo que requiere entonces de la relación entre los hombres a través del lenguaje (universitas lógon politikón).

El logos es lenguaje, palabra, pensamiento, obra. Así, la politeia, en tanto relación de los hombres entre sí y del hombre consigo mismo, se puede concebir como gobierno de sí, pero no para sí (en tanto interés propio), sino 
para los demás (interés público). Su gobierno ha de anteponer las tendencias del bien propio exclusivo (individual) y albergar el bien común (colegiado), el de la comunidad universitaria.

Es así que la universidad como politeia tiene la potencia de configurar cuerpos colegiados decisorios de los acontecimientos que en la universidad ocurren, de aquellos que promueve e incluso de aquellos que llegan desde fuera de ella. La universidad en tanto politeia tiene la potencia de movilizar las estructuras internas de sí misma, pero, además, la posibilidad de movilizar la sociedad en su conjunto, de cambiar incluso su estructura alrededor de los saberes que se van configurando y legitimando. Véase cómo desde su origen europeo ayuda a constituir un nuevo orden social:

Las ciudades dejan de lado la organización feudal en órdenes (oratores, bellatores, laboratores) y se comienzan a constituir como un modelo social, económico, político y cultural, con base en los oficios. Uno de estos oficios es el saber. Por eso al surgir, la universidad es el tercer poder medieval frente al Sacerdotium (el Papado) y el Regnum (príncipes y emperadores). Su función va a ser la preparación de profesionales sabios (teólogos, abogados, canonistas, médicos...) que con su saber cumplan una función clave en la estructuración de los saberes mismos y de la sociedad (Soto, 2007, p. 404).

La universidad en tanto politeia implica además la condición de espacio público, donde tiene ocasión la vida pública, el debate, la creación de ciudad, de profesionales que harán parte de la vida social y de las funciones propias que cada uno desempeñará en el Estado, en la comunidad, como ejercicio de configuración de lo público, no solo como escenario de lo público. Es decir, la universidad ha de cumplir la función de formar lo público, no solo de servir de escena de lo público o formar a los funcionarios públicos y privados según las profesiones necesarias para cada sociedad; formar lo público he ahí el sentido de su politeia, capaz de configurar comunidad universitaria, comunidad social.

"Los hombres se organizan políticamente según determinadas comunidades esenciales en un caos absoluto" (Arendt, 1997, p. 45). Históricamente la universidad es dentro de una sociedad una comunidad esencial, ya que permite erigirse como una institución que establece relaciones entre el saber y el poder, esto denota un carácter de ordenamiento y ascenso de las sociedades en virtud de los cuerpos de profesionales formados, capaces de asumir los destinos de las comunidades, de las sociedades, cada vez con mayores exigencias formativas institucionalizadas. 
Pero al mismo tiempo que se forman profesionales para asumir los destinos de las sociedades, se configuran formas políticas de relacionamiento que influyen en las estructuras de las universidades y de las sociedades mismas. De ahí que la política se establece a partir de relaciones entre los hombres y, según sean estas relaciones, se vive humanamente:

Recuérdese que fueron la Universidad (y la Iglesia) quienes al finalizar la segunda guerra mundial reconstruyeron las ciudades del centro de Europa (Lovaina, Dresde, Colonia...) y que hoy, en este contexto del profesor universitario, es llamado a reconstruir la universidad desde adentro, asumiendo la voz kantiana en tanto que toda comunidad primero ha de ser ética, para luego poder hacer ciencia y, por supuesto, alcanzar la autonomía en tanto creadora (la universidad poiética), formadora de la identidad ciudadana, intelectual, cultural y profesional, capaz de movilización social (universidad politeia), responsable del destino espiritual, social y material, en tanto que la universidad es la síntesis de cada pueblo (Pérez y Ríos, 2017b, p. 21).

La universidad -entre poiesis y politeia - es el eco de una voz olvidada (silenciada), que convoca con su llamado a la comprensión de la universidad como creación humana, pero a la vez como creadora. No se trata de la simple cuestión de producir profesionales, es más que esto. La universidad tiene la potencia de crear incluso de las cenizas y las ruinas a las sociedades mismas, es capaz de recrearlas, de ahí su potencia poiética-politéica. Dicha potencia permite movilizar las estructuras sociales, así como ha de movilizarse ella misma en sus estructuras internas, con la responsabilidad destinal de que no solo es el espacio donde ha de circular lo público, sino donde se ha de formar lo público. Las universidades actuales son la síntesis de cada pueblo, lo que implica pensar en el tipo de pueblo que somos capaces de ser, ya que esto determina el tipo de universidades que nosotros mismos somos capaces de constituir. Hemos de detenernos a pensar en esto y actuar en virtud de lo anterior, pues toda universidad inicia en el fondo como una actitud interior del ser humano y termina allí mismo.

\section{Tres consideraciones finales}

La primera consideración presenta la problematización de un doble origen de la universidad: uno histórico y otro erótico-creativo. El origen histórico se disputa tres lugares de enunciación: medieval, clásico y ancestral, cada uno de estos en relación con dos perspectivas de comprensión que se contraponen: una moderna, que se evidencia en Rashdall (1895, p. 146) y Ureña (1919, p. 
61); otra transmoderna, como la proponen Ribeiro (1971, p. 77) y Dussel (2006, p. 36). El otro origen de la universidad se plantea en perspectiva de creación humana (Kant, 1991, p. 3) y de acontecimiento interior de cultivo de la virtud y el conocimiento (Platón, 1999, p. 31), que da vida a la creación erótica, la cual es previa a todo acto de institucionalización.

Una segunda consideración se posa sobre la necesaria recuperación de la idea de universidad en nuestro siglo XXI. Esto es, al mismo tiempo, su propia problematización. Es la posibilidad de superar su doble olvido reconociendo el otro origen de la universidad (erótico-creativo-íntimo, más allá de las diversas descripciones históricas y de comprenderla como síntesis de los pueblos, como institucionalización). Por lo tanto, resulta imperativo actualizar la pregunta por la idea de universidad en una época en la que se ha naturalizado su desplazamiento y reemplazo por instituciones de educación superior en las que queda diluida su naturaleza y, con ella, su idea de formación originaria.

La tercera consideración problematiza la potencia politéica que tiene la universidad, en tanto posibilidad de movilización social y formación de lo público, no solo de circulación de lo público. La universidad se juega entre la reproducción social, prolongación de las brechas y desigualdades sociales y la transformación social. Así, la contingencia de un nuevo ordenamiento social se da mediante el acceso a la formación, el cultivo de sí mismo (autoformación) y la recuperación de la pregunta que indaga por el sentido de la universidad en la escolarización y profesionalización de la sociedad, en cuanto posibilitadora de procesos de transformación como horizonte capaz de alcanzar niveles superiores de lo humano.

De esta manera, la universidad se plantea como el esfuerzo espiritual y colectivo más grande de la humanidad por alcanzarse a sí misma, que ha de ser recuperado en medio de su desplazamiento silencioso e inadvertido por instituciones de educación superior que olvidan y borran su tradición, diálogo y vinculación con la filosofía. Llegados a este punto, conviene evocar la sentencia del profesor O. Pulido: "la educación ha de asumir la filosofía, en otras palabras, debe ser filosófica, en todas las áreas del saber incluida la misma Filosofía, pues no puede existir una educación para el pensamiento sin que haya actitud de filosofar" $(2012$, p. 98). 


\section{Referencias}

Adorno, T. W. (1959). Theorie der Halbbildung. Gesammelte Schriften Band 8, Soziologischen Schriften I (pp. 93-121). Darmstadt: Wissenschaftliche Buchgesellschaft.

Arendt, H. (1993). La condición humana. (Trad. R. Gil Novales). Barcelona: Paidós.

Aristóteles. (1873). Política. (Trad. P. de Azcárate). Madrid: Medina y Navarro.

Benjamin, W. (1991). Para una crítica de la violencia. (Trad. R. J. Blatt Weinstein). Madrid: Taurus.

Dussel, E. (2006). 20 tesis de politica. México: Siglo XXI.

Foucault, M. (2015). ¿Qué es la Ilustración? (Trad. A Gabilondo Pujol). Medellín: Universidad de Antioquia.

Hincapié, A. (2016). Formación y praxis pedagógica revolucionaria: los escritos de juventud de Marx y Benjamin. Andamios, 13(32), pp. 257-279.

Kant, I. (1991). Antropología en sentido pragmático. (Trad. J. Gaos). Madrid: Alianza Editorial.

Naishtat, F., García, A., y Villavicencio, S. (2001). La universidad hoy: crisis de "esa buena idea". F. Naishtat, A. García y S. Villavicencio (Comps.), Filosofías de la universidad y conflicto de racionalidades (pp. 19-32). Buenos Aires: Colihue.

Nietzsche, F. (1872/2000). El porvenir de nuestras instituciones educativas. (Trad. Carlos Manzano). Madrid: Alianza Editorial.

Platón. (1993). La República. (Trad. José Manuel Pabón y Manuel Fernández Álvarez). Madrid: Ediciones Altaya S.A.

Platón. (1999). El Simposium. (Trad. Patricio de Azcárate). Madrid: Ediciones Altaya S.A.

Pérez, F., Ríos, E., Valencia, W., y Salazar, A. (2015). Orientaciones para el maestro de humanismos. Rionegro: Fondo Editorial Universidad Católica de Oriente. 
Pérez, F., y Ríos, E. (2017a). Consideraciones sobre la universidad, la formación profesional y el maestro universitario. Editor: Julio Cesar Arboleda Aparicio. Compiladores: Mendoza, W; Rengifo, C; Arias, J. Pedagogías Críticas (pp. 84-93). Cali: Editorial Bonaventuriana y REDIPE.

Pérez, F., y Ríos, E. (2017b). La Universidad y El Profesor Universitario. (Documento de estudio). Medellín: Asociación Colombiana de Universidades (ASCUN) y Asociación Colombiana de Facultades de Educación (ASCOFADE).

Pulido, O. (2012). Estanislao Zuleta: educación con filosofía. Cuestiones de Filosofía, 14, pp. 79-99.

Rashdall, H. (1895). The Universities of Europe in the Middle Ages. Oxford: Clarendon Press.

Restrepo, C. (2013). La destrucción de la universidad. Autonomía y éxodo del conocimiento hacia la universidad nómada. L. A. Castrillón López (Dir.), La universidad por hacer. Perspectivas poshumanistas para tiempos de crisis (pp. 85-101) Medellín-Rionegro: Universidad Pontificia Bolivariana y Universidad Católica de Oriente.

Ribeiro, D. (1971). La universidad latinoamericana. Caracas: Universidad Central de Venezuela.

Soto, G. (2007). Filosofía Medieval. Bogotá: Ediciones San Pablo y Universidad Pedagógica Nacional.

Ureña, P. (1919). La Universidad. México: UNAM. 